\title{
How Should We Be Conducting Routine Analysis of Traditional Emergency Department Syndromic Surveillance Data?
}

\author{
David Atrubin $^{\star 2}$ and Michael Wiese ${ }^{1}$ \\ ${ }^{1}$ FL DOH - Hillsborough Cnty, Tampa, FL, USA; ${ }^{2}$ Florida Department of Health, Tampa, FL, USA
}

\section{Objective}

To discuss how various emergency department based syndromic surveillance systems from across the country and world are being used and to develop best practices for moving forward

\section{Introduction}

Along with commensurate funding, an increased emphasis on syndromic surveillance systems occurred post September 11, 2001 and the subsequent anthrax attacks. Since then, many syndromic surveillance systems have evolved and have ever-increasing functionality and visualization tools. As outbreak detection using these systems has demonstrated an equivocal track record, epidemiologists have sought out other interesting and unique uses for these systems. Over the numerous years of the International Society for Disease Surveillance (ISDS) conference, many of these studies have been presented, however, there has been a dearth of discussion related to how these systems should be used on a routine basis. As the initial goal of these systems was to provide a near real-time disease surveillance tool, the question of how to most effectively conduct this type of routine surveillance is paramount.

\section{Description}

This roundtable will provide a forum for national, state, and local users of syndromic surveillance systems to discuss how they use these systems routinely. With the increasing number of participating hospitals (due in large part to increased incentives from Meaningful Use), this question has taken on added significance. An important part of this discussion will focus on the value that syndromic surveillance can provide that other surveillance systems cannot address.

\section{Audience Engagement}

This roundtable is well suited to audience participation as the discussion will revolve around how different jurisdictions are utilizing their syndromic surveillance data. Roundtable participants will be asked about their routine surveillance methods, including the frequency of data analysis and the type of reports that are produced. Participants will also be encouraged to share their opinions as to uses and misuses of syndromic surveillance data sets.

\section{Sample Questions:}

-How does your jurisdiction conduct syndromic surveillance each day/periodically?

-Does syndromic surveillance need to be conducted locally or is it possible to do it effectively at the state of national level?

-When conducting an epidemiologic analysis, how should decisions be made regarding using a syndromic surveillance data set versus a hospital discharge data set?

\section{Keywords}

emergency department data; routine analysis; Local public health; disease surveillance; Outbreak detection
*David Atrubin

E-mail: david.atrubin@flhealth.gov 